\title{
Aplicación del Aprendizaje Basado en Retos en el primer curso del Grado de Geografia y gestión del Territorio
}

\section{Application of Challenge-Based Learning in the first-year course of the Degree in Geography and Territorial Management}

DAVID SÁNCHEZ BENÍTEZ

ORCID: https://orcid.org/0000-0001-9581-2981

Universidad de Sevilla

Departamento de Geografia Humana

dsanchez5@us.es

Fecha de recepción: 19/11/2019

Fecha de revisión: 21/11/2019

DOI: http://dx.doi.org/10.12795/9788447221912.067

Pp.: 1546-1568 
Resumen

Se presentan en este artículo los resultados de la experiencia de innovación docente dentro de los CIMA (Ciclos de Mejora en el Aula) desarrollada en la REFID (Red para la Formación y la Innovación Docente) de la Universidad de Sevilla durante el primer cuatrimestre del curso 2019/2020. Se abordaron los contenidos pertenecientes al tema Investigación y docencia en el campo de la Geografía Humana del bloque Geografía Humana Aplicada de la asignatura Geografía Humana de primero de Grado en Geografia y Gestión del Territorio. La innovación se centró en la aplicación del Aprendizaje Basado en Retos (ABR) a partir de la metodología de Pensamiento de Diseño (Design Thinking, DT) adaptada como ECO trabajando escenarios lo más cercanos posible a la realidad del alumnado y a sus futuras salidas profesionales.

Palabras clave: Geografía Humana, docencia universitaria, innovación, Design Thinking, Aprendizaje Basado en Retos.

\section{Abstract}

This article is based on the results of the teaching innovation experience within the CIMA (Ciclos de Mejora en el Aula) in the REFID (Red para la Formación y la Innovación Docente) at Universidad de Sevilla during the first semester of the academic year 2019/2020. The contents belongs to the unit Research and teaching in Human Geography of the Applied Human Geography contents of the subject Human Geography of the Degree in Geography and Territorial Management. The innovation is focused on the application of Challenge-Based Learning with the methodology Design Thinking adapted to ECO working with scenarios as close as possible to the reality of students and their future career opportunities. .

Key words: Human Geography, Higher Education, Innovation, Design Thinking, Challenge-Based Learning.

Jornadas de Formación e Innovación Docente del Profesorado | № 2 (2019) Esta obra se distribuye con la licencia Creative Commons Reconocimiento-NoComercial-SinObraDerivada 4.0 Internacional (CC BY-NC-ND 4.0.) 


\section{Contexto}

La asignatura con la que se ha trabajado la Fase de Permanencia de la Red para la Formación y la Innovación Docente (REFID) del Programa de Formación e Innovación Docente del Profesorado de la Universidad de Sevilla en el marco del III Plan Propio de Docencia durante el primer cuatrimestre del curso 2019/2020 es la llamada Geografia Humana. Se trata de una asignatura obligatoria de primer cuatrimestre de primer curso del Grado en Geografia y Gestión del Territorio. El Ciclo de Mejora en el Aula (en adelante CIMA) se llevó a cabo en el grupo de tarde con horario de clase los lunes y martes de 17.00 a 18.30 horas.

Los 6 créditos de la asignatura se encuentran repartidos en 4,5 en teórico-prácticos y 1,5 de prácticas de campo, de los que me encargo exclusivamente de los teórico-prácticos. Esta circunstancia supone un cambio respecto a las anteriores CIMA realizados que se aplicaron en otra asignatura y con la posibilidad de contar con el tiempo reservado a los créditos de prácticas de campo. Este cambio supuso una limitación que se sumó a la circunstancia de que ha sido el primer curso en el que me he encargado de la docencia de esta asignatura.

En relación al alumnado, el número de personas matriculadas era de 30, oscilando el número de asistentes a las clases durante todo el cuatrimestre entre los 20 y 25, un número que permitía, mediante la formación de pequeños grupos llevar a cabo esta experiencia docente.

El aula asignada para la docencia de la asignatura no se prestaba especialmente a la experiencia al estar amueblada con 9 filas de bancas corridas fijadas al suelo de 7 asientos, sin pasillo intermedio, pequeños pasillos laterales, escasa luz natural, una única pared que utilizar como soporte para visualizar materiales y problemas de recepción de la red wifi. 
El objetivo del CIMA fue trabajar profesorado y alumnado a través del Aprendizaje Basado en Retos (ABR) con metodología de Pensamiento de Diseño o DT (Design Thinking), para cambiar el enfoque de la enseñanza al aprendizaje en una asignatura de primero. El alumnado en el primer cuatrimestre del Grado se enfrenta a multitud de conceptos nuevos de bastante relevancia en materias de cursos superiores. Al ser muchos los temas tratados, se corre el riesgo de que puedan aproximarse a ellos como algo abstracto y lejano a su vida cotidiana. Se escogió esta metodología tras recibir formación en la misma en diversos cursos del Instituto de Ciencias de la Educación de la Universidad de Sevilla a cargo de Eduardo Herrero, Juan Jesús Torres y José Becerra.

\section{Diseño previo del Ciclo de Mejora Docente}

\section{Modelo metodológico.}

Como ya se ha comentado anteriormente, es el primer curso en el que me encargo de la docencia de los créditos teórico-prácticos de esta asignatura por lo que desconocía el modelo metodológico utilizado en cursos anteriores para el conjunto de la asignatura. En el programa de la asignatura vigente se resume la metodología de enseñanza-aprendizaje como clases en las que se combinan la exposición teórica con la realización de prácticas, exposiciones y debates, y el comentario y discusión de textos. Está previsto que en las sesiones teóricas el profesor plantee los conceptos más relevantes que después deben ser trabajados por el alumnado a través de la búsqueda de información, lectura crítica de diversos materiales y puesta en común en el aula y en la asignatura Taller Práctico de Geografia Humana, que se imparte en el segundo cuatrimestre. También se insta en el programa aprobado hasta 
el curso pasado a que el alumnado, con presencia del profesor, sea tutelado en la realización de actividades académicas dirigidas, siendo la labor del docente la asesoría, corrección y reconducción de las mismas.

Teniendo en cuenta todo lo anterior, un modelo ideal alternativo podría ser uno basado en el principio de investigación en el que un reto vaya conduciendo a los alumnos hacía la construcción del conocimiento. El papel de guía del profesor se mantiene, pero es el alumnado el protagonista de su propio aprendizaje (Bain, 2007) y el responsable de seleccionar un reto lo más cercano posible a su realidad e intereses. Mediante este reto, el alumnado podría ir trabajando diferentes aspectos del temario a través de esos conceptos básicos comentados anteriormente y con los que pueden acercase a los diferentes bloques. Además, la matriculación de alumnos para el grupo de tarde (en este curso un total de 30 estudiantes), facilita la constitución de pequeños equipos en los que poder consensuar esos retos.

El modelo metodológico posible que he considerado viable realizar en este curso sería el citado modelo ideal pero aplicado solo a uno de los bloques de la asignatura. Se diseñó así un CIMA de carácter parcial de 14 horas de duración, que se desarrolló a lo largo de 12 horas presenciales de clase repartidas en diferentes sesiones. Dadas las características de la asignatura y al ser el primer año que la impartía, seleccioné el tema dedicado a las salidas profesionales del grado, último previsto en el programa.

La elección del citado tema conllevaba una serie de ventajas. En primer lugar, el tema se podía adelantar en el calendario programado y poner fácilmente en relación con el introductorio de la asignatura y con el relacionado con la ordenación del territorio y el urbanismo. El segundo aspecto a destacar está relacionado con la tipología y características del alumnado de primer curso. En un alto porcentaje, este se enfrenta con las primeras asignaturas de 
un grado que no ha elegido como primera opción y suele haber, a priori, falta de motivación e interés, y las salidas profesionales del mismo les suelen ser por lo general desconocidas.

Para acercar al alumnado a estos contenidos, el modelo docente empleado ha sido, por tanto, el Aprendizaje Basado en Retos (en adelante ABR) con metodología DT. El Pensamiento de Diseño es una metodología, aplicable al aula, para la resolución de problemas o desafios, ofreciendo soluciones que responden a las necesidades reales de la sociedad. Los resultados deben ser lo más creativos posible y los colectivos a los que se dirigen muy variados por lo que se fomenta la diversidad de enfoques y temas tratados en el aula. Además la metodología DT/ECO supone un cambio ya que la docencia no se centra solo en el docente y el alumnado y los contenidos, sino también en la sociedad y en sus necesidades. El alumnado tiene que detectar una necesidad de la sociedad para convertirla en un reto a través del cual acercarse a los contenidos de la asignatura y desarrollar una serie de ideas entre las cuales se encontrará la solución al reto que debe ser innovadora y original. Por tanto, los retos nacen de necesidades de la sociedad, desafios a los que se enfrentará el alumnado en su futuro profesional.

Más detalladamente, con esta metodología el alumnado debe pasar por tres etapas principales a partir de cuyas iniciales crearíamos el acrónimo ECO. La primera de ella es explora, es decir, identificar el reto a través del cuestionamiento en el propio grupo de sus inquietudes y de la posterior realización de entrevistas a los colectivos - las personas que pueden estar afectadas por las mismas inquietudes, además de investigar qué se sabe del tema (con el apoyo de los materiales de la asignatura a su disposición la enseñanza virtual). En una segunda fase el alumnado crea ideas para imaginar posibles soluciones y seleccionar la más innovadora y creativa según sus 
propios criterios (viabilidad, originalidad, etc.). Por último, deben pasar por una etapa en la que ofrecen esa solución mediante un prototipo. Posteriormente se debe recibir feedback de los compañeros y de ese colectivo social al que se pretende ayudar para mejorar ese prototipo y, por tanto, la solución al reto. Todo ello se va realizando a través de diferentes actividades de las que se va teniendo evidencia de su trabajo con diversos instrumentos.

En resumen, el ciclo de mejora engloba un tema completo de la asignatura Geografía Humana dedicado a la Investigación y docencia en el campo de la Geografia Humana dentro del bloque Geografia Humana aplicada y se ha realizado mediante el diseño de retos por el alumnado en equipos a partir de la metodología Aprendizaje Basado en Retos-Design Thinking/ECO para el que se detalla posteriormente la secuencia de actividades.

\section{Mapa de contenidos}

Para comenzar, es importante destacar que los contenidos de la asignatura seleccionada están centrados en conceptos básicos y actuales en el campo de la Geografía como el medio ambiente, la globalización, la innovación, o el desarrollo y cohesión territorial pero también dar a conocer la parte práctica y profesional de la Geografia Humana. Es decir, hablamos de una asignatura en la que se introducen por primera vez conceptos clave en posteriores asignaturas del ámbito de la Geografía Humana y que precede a una asignatura de segundo cuatrimestre de carácter más práctico.

Para llevar a cabo el ciclo de mejora se ha realizado una revisión de los contenidos del tema denominado Investigación y docencia en el campo de la Geografia Humana en el que se aborda la docencia en la enseñanza secundaria (ESO, Bachillerato) y universitaria (10 y 2o Ciclos 
y Doctorado) de la Geografía, la investigación científica en la Universidad o en otras instituciones y el trabajo en consultoras privadas y diferentes instituciones vinculadas a la administración pública regional y local. Los contenidos del tema están dirigidos a resolver una necesidad real; que conozcan las salidas profesionales del Grado de Geografia y Gestión del Territorio y la estructura de los contenidos de la Geografía Humana a lo largo del mismo para así poder orientar su formación en los siguientes cursos conforme a sus intereses. También se busca que conozcan nuevas líneas de estudio dentro de la Geografia y ponerlas en relación con su contexto social.

Por lo tanto, el contenido vertebrador del CIMA (ver figura 1) serían las líneas de trabajo de los geógrafos, reconociéndolas y relacionándolas con las salidas profesionales del grado y con las materias que trabajarán en futuros cursos. Se buscará la máxima relación con los contenidos vertebradores de los bloques anteriores (desigualdades, globalización, sostenibilidad) pero intentando que los retos no se focalicen en una división clásica de las salidas profesionales en investigación, docencia y consultoría $y$, a su vez, que relacionen esas líneas de estudios y salidas con la realidad de su entorno y las desigualdades sociales del mismo.

Por último, es importante matizar que este unidad se encuentra relacionada con la anterior del temario titulada La Geografia Humana y el planeamiento espacial y sectorial en la que se trabajan la incorporación de la Geografía Humana al planeamiento espacial, los conceptos de ordenación del territorio y urbanismo y la aportación de la Geografia Humana al planeamiento sectorial (agrario, medioambiental, industrial...) que suponen, asimismo, salidas laborales del profesional de la Geografia. 


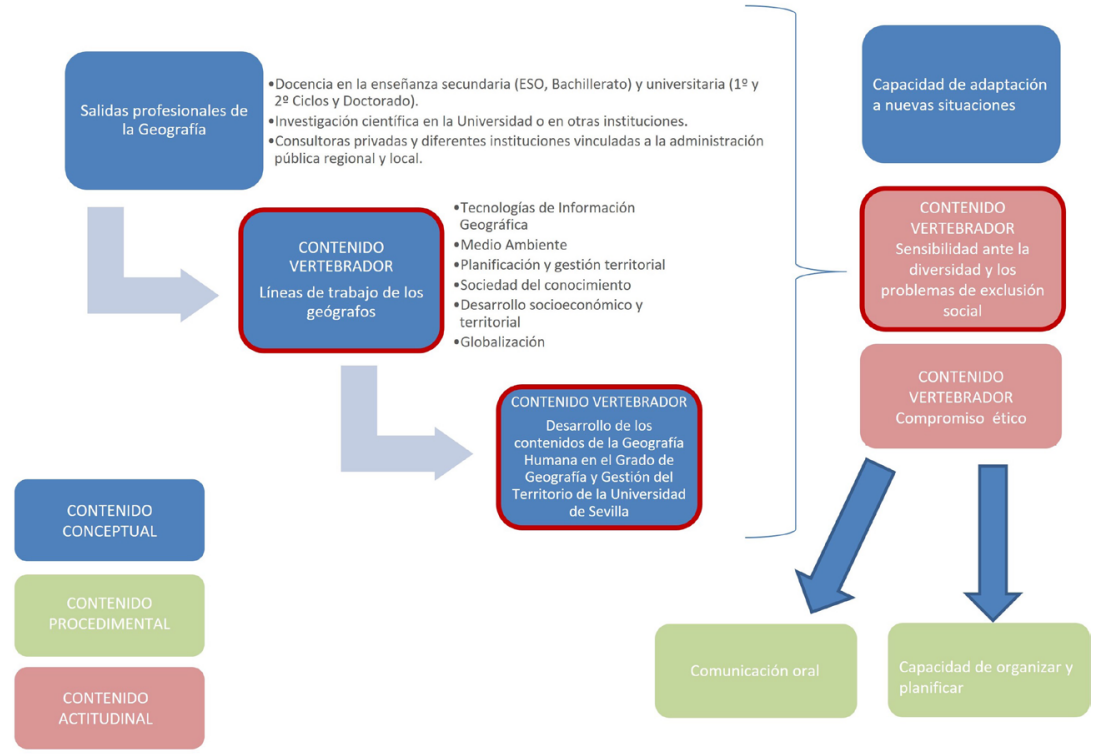

Figura 1. Mapa de contenidos del tema "Investigación y docencia en el campo de la Geografía Humana".

\section{Secuencia de actividades}

El ciclo de mejora se desarrolló a partir de la siguiente secuencia de sesiones y actividades en las que se fue realizando un seguimiento en clase del trabajo realizado por el alumnado:

Tabla 1 Cronograma de actividades desarrollado en el CIMA

\begin{tabular}{|l|l|l|}
\hline Sesión & Actividad & Descripción \\
\hline $\begin{array}{l}\mathbf{1} \\
\text { (1 hora) }\end{array}$ & $\mathbf{1}$ & $\begin{array}{l}\text { Entrega del cuestionario previo en el que } \\
\text { se valora través de diferentes niveles de } \\
\text { aprendizaje para los contenidos seleccionados } \\
\text { el nivel de partida del alumnado (ver tabla 2). }\end{array}$ \\
\cline { 2 - 3 } & $\mathbf{2}$ & $\begin{array}{l}\text { Presentación de la estructura del ciclo en clase, } \\
\text { formación de grupos por intereses y asignación } \\
\text { de roles. }\end{array}$ \\
\hline
\end{tabular}

Jornadas de Formación e Innovación Docente del Profesorado | № 2 (2019) Esta obra se distribuye con la licencia Creative Commons Reconocimiento-NoComercial-SinObraDerivada 4.0 Internacional (CC BY-NC-ND 4.0.) 


\begin{tabular}{|c|c|c|}
\hline $\begin{array}{l}2 \\
\text { (1 hora) }\end{array}$ & 1 & $\begin{array}{l}\text { Planteamiento del problema. Relación las ideas } \\
\text { y conocimientos ya adquiridos por el alumnado } \\
\text { y sus emociones, para que, ya organizados } \\
\text { en equipos, detecten una necesidad de la } \\
\text { sociedad relacionada con los contenidos del } \\
\text { tema (qué se investiga y enseña en Geografia) } \\
\text { y la asignatura (contenidos vertebradores de } \\
\text { otros bloques de la materia centrada en las } \\
\text { desigualdades, las desequilibrios económicos } \\
\text { o poblacionales, etc) y los colectivos afectados } \\
\text { por el mismo para convertir esa necesidad en } \\
\text { un reto. }\end{array}$ \\
\hline $\begin{array}{l}3 \\
\text { (1 hora) }\end{array}$ & 1 & $\begin{array}{l}\text { Definir, concretar el reto. Tras esa puesta en } \\
\text { común de inquietudes personales se realizará } \\
\text { una actividad mediante la cual redactarán un } \\
\text { pequeño guion de preguntas abiertas para } \\
\text { detectar esas necesidades en sus ámbitos } \\
\text { familiar y de estudio y poder centrar el reto en } \\
\text { un colectivo o inquietud determinados. }\end{array}$ \\
\hline $\begin{array}{l}4 \\
\text { (4 horas) }\end{array}$ & 1 & $\begin{array}{l}\text { Sesión fuera del aula para recoger información, } \\
\text { realización de entrevistas, fotografias, etc } \\
\text { y puesta en común del material recopilado } \\
\text { mediante la enseñanza virtual y la herramienta } \\
\text { padlet. }\end{array}$ \\
\hline $\begin{array}{l}5 \\
\text { (1 hora) }\end{array}$ & 1 & $\begin{array}{l}\text { Puesta en común del reto y del conocimiento } \\
\text { y contenidos de la materia que pueden servir } \\
\text { para llegar a ideas innovadoras que puedan } \\
\text { concretarse en una solución original. Se } \\
\text { trabajarán competencias relacionadas con la } \\
\text { búsqueda de información bibliográfica. }\end{array}$ \\
\hline $\begin{array}{l}6 \\
\text { (1 hora) }\end{array}$ & 1 & $\begin{array}{l}\text { Puesta en común de ideas: posibles soluciones. } \\
\text { Encontrar la más efectiva, creativa y viable. }\end{array}$ \\
\hline $\begin{array}{l}7 \\
\text { (1 hora) }\end{array}$ & 1 & $\begin{array}{l}\text { Puesta en común de los prototipos: } \\
\text { colaboración entre los grupos para evaluar, } \\
\text { probar el modelo: la solución identificada como } \\
\text { las más eficaz y original. }\end{array}$ \\
\hline $\begin{array}{l}8 y 9 \\
\text { (3 horas) }\end{array}$ & 1 & $\begin{array}{l}\text { Ofrecer a la sociedad la solución al reto, es } \\
\text { decir, presentación por grupos en el aula de } \\
\text { las materiales generados por cada grupo a } \\
\text { través del portavoz y siempre con el alumnado } \\
\text { en primer plano y el profesor actuando como } \\
\text { guía de las soluciones a los retos (póster, vídeo, } \\
\text { webs, maquetas, etc). }\end{array}$ \\
\hline
\end{tabular}




\begin{tabular}{|l|l|l|}
\hline $\begin{array}{l}\text { (1) hora) } \\
\text { (1 }\end{array}$ & $\begin{array}{l}\text { Debate final. Se intenta en esta fase utilizar } \\
\text { las técnicas apropiadas para generar un } \\
\text { aprendizaje crítico y la mayor relación posible } \\
\text { con los contenidos de otros temas de la } \\
\text { asignatura, dando validez a las distintas } \\
\text { respuestas generadas al problema en los } \\
\text { diferentes grupos. }\end{array}$ \\
\cline { 2 - 3 } & $\begin{array}{l}\text { Entrega el mismo cuestionario utilizado de } \\
\text { manera previa al taller para valorar los cambios } \\
\text { en los conocimientos del alumnado para cada } \\
\text { contenido vertebrador asi como el diseñado } \\
\text { para ayudar en la evaluación del modelo con } \\
\text { las preguntas de la tabla } 2 .\end{array}$ \\
\hline
\end{tabular}

\section{Cuestionario inicial-final}

Deben valorarse los conocimientos iniciales y posteriores a la resolución del reto fijado por cada grupo para observar los cambios en su conocimiento. Para ello se realizó un cuestionario en el que pudiesen aparecer reflejados algunos de los contenidos más conceptuales del tema en sus primeras preguntas, y que preguntase por alguno de los contenidos actitudinales en la última (ver tabla 2). En el mismo, a través de diferentes niveles de aprendizaje que se localizaron para los contenidos seleccionados se valoró el nivel de partida del alumnado, y al ser el mismo cuestionario utilizado para la postevaluación, también el alcanzado tras el CIMA. El cuestionario es lo suficientemente poco específico para que puedan caber en el las respuestas diversas de un alumnado que ha guiado su proceso de aprendizaje a partir de retos diferentes siguiendo sus inquietudes.

Tabla 2: Preguntas cuestionario pre-post evaluación

\section{PREGUNTAS CUESTIONARIO PRE-POST EVALUACIÓN}

¿Qué campos tiene la Geografía?

¿Qué palabras relacionáis con la Geografía Humana?

Jornadas de Formación e Innovación Docente del Profesorado | № 2 (2019) Esta obra se distribuye con la licencia Creative Commons Reconocimiento-NoComercial-SinObraDerivada 
¿Qué salidas profesionales tiene la Geografia?

¿Cómo podréis como geógrafas/os colaborar con vuestro entorno?

Así mismo, se valorará el trabajo realizado como parte de la calificación de la asignatura con un porcentaje de un $15 \%$ para el alumnado que sigue la evaluación continua. Se trata de un porcentaje en la calificación que representa el peso del bloque y supone un aliciente. Para todo ello, se tendrá en cuenta no solo la evolución y obstáculos detectados en el aprendizaje mediante los cuestionarios citados sino también el trabajo realizado en las sesiones y la exposición del prototipo en la sesión final.

También se realizó una evaluación del diseño y desarrollo del CIMA por parte del alumnado mediante cuestionarios (ver tabla 3) que se sumó a todo lo anotado para el seguimiento del proceso de enseñanza-aprendizaje por parte del docente en un diario de las sesiones.

Tabla 3: Preguntas cuestionario modelo de aprendizaje

PREGUNTAS CUESTIONARIO MODELO DE APRENDIZAJE

¿Qué te ha gustado más de la metodología llevada a cabo en este trabajo y por qué?

¿Qué mejorarías y por qué?

Si tuvieras que explicar a tus compañeros cómo es la investigación y

docencia en el campo de la Geografía Humana, ¿cómo lo harías?

\section{Aplicación del CIMA}

\section{Relato resumido de las sesiones}

Las sesiones programadas se desarrollaron como estaba previsto, siendo la primera de las dificultades a la que hacer frente las continuas incorporaciones de alumnado a lo largo de las primeras semanas de clase por el 
proceso de adjudicaciones de plazas de septiembre y ser el Grado de Geografia y Gestión del Territorio uno de los que mantiene oferta de plazas para esta convocatoria. No fue hasta la cuarta semana de clases del cuatrimestre que no se estabilizó el número de asistentes a las sesiones y hubo que ir reorganizando los grupos haciendo desaparecer alguno y creando otros nuevos.

Así mismo habría que destacar como suceso no esperado el porcentaje de alumnado de otras nacionalidades, 7 alumnos extranjeros de los 24 alumnos que acuden de manera asidua a clase (de nacionalidad estadounidense en cinco de los casos más dos alumnas Erasmus procedentes de Bélgica e Italia). Desgraciadamente, debido a que el alumnado estadounidense finalizaba sus programas la última semana de diciembre fue imposible por motivos de evaluación que estuviesen dispersos por los diferentes grupos y acabaron formando dos equipos.

Para garantizar que el alumnado profundizara en los retos de los demás y para que se fueran cubriendo todos los ámbitos del bloque de Geografía Humana Aplicada, y en especial del tema Investigación y docencia en el campo de la Geografia Humana, se agruparon por afinidades e inquietudes profesionales formando un total de 7 grupos compuestos por una media de 3 personas y centrados en el papel del profesional de la Geografia en la docencia de la Geografía (3 grupos), la investigación y la difusión del conocimiento (3 grupos) y la Ordenación del Territorio y el Urbanismo (1 grupo). De manera previa a la formación de grupos se realizó una actividad no prevista mediante la cual identificaban de una serie de fotografias aquella que simbolizaba más cómo estaban viviendo las primeras semanas de experiencia universitaria, para debatir sobre cuáles eran sus expectativas sobre la formación universitaria. También visualizamos dónde querrían trabajar al acabar el grado o su formación mediante una gráfica que creamos con la aplicación mentemeter y el uso del móvil. 
En la sesión dedicada al planteamiento del problema se trabajó la relación de las ideas y conocimientos ya adquiridos por el alumnado y sus emociones, a partir del debate surgido a con de la pregunta ¿Qué palabras relacionáis con la Geografia Humana? y la nube de palabras generada con sus repuestas de nuevo con la herramienta mentemeter. Ya organizados en equipos, centraron el debate en sus inquietudes y trataron de localizar una necesidad de la sociedad relacionada con el marco de actuación de cada grupo (aplicaciones de las personas graduadas en Geografia, en la docencia, la investigación o la ordenación del territorio) y en temas relacionados con el conjunto de la asignatura (las desigualdades, los desequilibrios económicos o poblacionales, etc) y los colectivos afectados por el mismo para convertir esa necesidad en un reto. De manera previa se realizó también una actividad con extractos de textos de nuevos estudios dentro de diversas aplicaciones en el Campo de la Geografia Humana al que tenían que nombrar como una supuesta nueva subdisciplina geográfica.

En la siguiente sesión tras una puesta en común de inquietudes personales crearon un breve guion de tres preguntas para la entrevista que realizarían en la siguiente sesión a representantes de colectivos implicados en su marco de acción. Antes de la realización de la entrevista realizamos una pequeña actividad para acercarnos al concepto de empatía.

Los resultados de esa entrevistas quedaron plasmados en una pizarra común mediante la herramienta padlet en la que se recogían para cada grupo el guion de preguntas, las repuestas de las personas entrevistadas (con las transcripción de las respuestas o los audios de la entrevistas) y una fotografia en formato selfie con el grupo. Esto facilitó la puesta en común en clase y la posibilidad de intercambiar experiencias e información entre los grupos.

En la siguiente sesión expusieron el reto escogido al resto de compañeros a partir de toda la información 
recogida y se presentaron los contenidos del bloque de Geografia Humana Aplicada a su disposición en la enseñanza virtual. También revisamos un amplio directorio de enlaces y materiales (planes de grados, máster, enlaces a asociaciones y colegios profesionales) y se trabajaron competencias relacionadas con la búsqueda de información bibliográfica a partir de un pequeño taller de búsqueda de información y elaboración de citas y referencias bibliográficas. Como ejemplo, en esta sesión un grupo de alumnas que trabajaba la relación teórico-práctica de los contenidos de la asignatura del grado expuso como habían cambiado la orientación del reto de una aparente desproporción a favor de los de contenidos prácticos para ellas como alumnas de primero a una falta de base teórica tras las entrevistas realizadas a alumnado de cursos superiores. El resto de grupos centraron sus retos en la mejora del papel social de la Geografía y la comunicación y participación social en la elaboración de documentos como los Planes Generales de Ordenación Urbana o las investigaciones desarrolladas en torno a efectos del cambio climático o el consumo de recursos a nivel local.

En una sesión posterior identificaron individualmente posibles ideas-solución al reto, las pusieron en común y seleccionaron 3 a las que aplicaron 3 criterios de idoneidad escogidos por cada grupo (efectividad, creatividad y viabilidad, coste, etc.). Todo ello quedo recogido en una matriz resumen de las ideas y los criterios por grupo que entregaron tras finalizar la sesión.

Por último, en una última sesión, la puesta en común de la primera versión de los prototipos sirvió para que entre los grupos evaluasen y colaborasen en la idoneidad de la solución seleccionada como las más eficaz y original tras la presentación en el aula de las materiales generados por cada grupo a través de un portavoz. A pesar de haber ido poniendo en común los resultados de cada uno de los pasos anteriores, no fue hasta esta presentación cuando se produjo más interacción entre los grupos y cuando varios de los grupos localizaron problemas en 
la identificación del reto o la elección de las soluciones o los criterios utilizados y decidieron reconducir sus retos. El resto de los grupos defendió sus prototipos (aunque en algunos casos solo expusieron la solución al reto sin apoyo de ningún recurso físico) y obtuvieron feedback del resto para realizar una versión más desarrollada del prototipo o complementar su idea con soluciones más originales o útiles al colectivo al que se dirigían los retos.

Se distribuyó el mismo cuestionario utilizado de manera previa al taller para valorar los cambios en los conocimientos del alumnado para cada contenido vertebrador, así como el diseñado para ayudar en la evaluación del modelo con las preguntas de la tabla 2. A este último cuestionario respondieron también con una respuesta consensuada dentro del grupo sobre los aspectos a mantener, a cambiar o a introducir al final de la sesión.

\section{Evaluación del aprendizaje del alumnado}

El sistema de evaluación del aprendizaje utilizado fue el de las escaleras de aprendizaje, para conocer el punto de partida y final de los alumnos. Volviendo a los contenidos que aparecen en la figura 1, se seleccionaron los siguientes para la elaboración de los cuestionarios que debieron rellenar antes y después del CIMA:

- Contenidos de la Geografía Humana en el Grado (preguntas 1 y 2 de la tabla 2).

- Líneas de trabajos de las personas graduadas en Geografía (pregunta 3 de la tabla 2).

- Sensibilidad ante la diversidad de problemas de exclusión social (pregunta 3 de la tabla 2).

Al objeto de elaborar escaleras de aprendizaje fundamentadas se han tomado las respuestas del alumnado que completó el cuestionario tanto en la fase inicial del taller como en la final. De este modo, trabajamos con un total de 22 cuestionarios. 
A continuación se enumeran las preguntas del cuestionario, los posibles niveles de conocimiento según sus respuestas en la escalera de aprendizaje y el porcentaje de cuestionarios en cada una de los escalones antes y después de las sesiones.

Tabla 4. Preguntas 1 y 2 del cuestionario y niveles de aprendizaje: ¿Qué campos tiene la Geografia?/¿Qué palabras relacionáis con la Geografia Humana?

\begin{tabular}{|l|l|l|}
\hline $\begin{array}{l}\text { Nivel de } \\
\text { respuesta }\end{array}$ & Descripción & $\begin{array}{l}\text { Porcentaje de } \\
\text { respuestas }\end{array}$ \\
\hline Nivel 1 & $\begin{array}{l}\text { No acierta a diferenciar entre Geografia Física } \\
\text { y Humana. Utiliza descriptores sin sentido o } \\
\text { imprecisos. }\end{array}$ & $\begin{array}{l}\text { Pre: 58\% } \\
\text { Post: 9\% }\end{array}$ \\
\hline Nivel 2 & $\begin{array}{l}\text { Diferencia entre Geografia Física y Humana y } \\
\text { apunta algunos descriptores correctos. }\end{array}$ & $\begin{array}{l}\text { Pre: 33\% } \\
\text { Post: 28\% }\end{array}$ \\
\hline Nivel 3 & $\begin{array}{l}\text { Diferencia entre Geografia Física y Humana y } \\
\text { apunta bastantes descriptores correctos. }\end{array}$ & $\begin{array}{l}\text { Pre: 9\% } \\
\text { Post: 45\% }\end{array}$ \\
\hline Nivel 4 & $\begin{array}{l}\text { Diferencia entre Geografia Física y Humana } \\
\text { y es capaz de subdividir en disciplinas sus } \\
\text { contenidos o conceptos integradores. }\end{array}$ & $\begin{array}{l}\text { Pre: 0\% } \\
\text { Post: 18\% }\end{array}$ \\
\hline
\end{tabular}

Tabla 5. Pregunta 3 del cuestionario y niveles de aprendizaje: ¿Qué salidas profesionales tiene la Geografia?

\begin{tabular}{|l|l|l|}
\hline $\begin{array}{l}\text { Nivel de } \\
\text { respuesta }\end{array}$ & Descripción & $\begin{array}{l}\text { Porcentaje de } \\
\text { respuestas }\end{array}$ \\
\hline Nivel 1 & No conoce ninguna & $\begin{array}{l}\text { Pre: 38\% } \\
\text { Post: 0\% }\end{array}$ \\
\hline Nivel 2 & $\begin{array}{l}\text { Nombra alguna pero con lenguaje poco } \\
\text { académico }\end{array}$ & $\begin{array}{l}\text { Pre: 37\% } \\
\text { Post: 23\% }\end{array}$ \\
\hline Nivel 3 & $\begin{array}{l}\text { Identifica las principales salidas } \\
\text { profesionales aunque sin utilizar un lenguaje } \\
\text { académico. }\end{array}$ & $\begin{array}{l}\text { Pre: 25\% } \\
\text { Post: 68\% }\end{array}$ \\
\hline Nivel $\mathbf{4}$ & $\begin{array}{l}\text { Identifica las principales salidas } \\
\text { profesionales, las valora o indica su reflejo } \\
\text { en la estructura del Grado }\end{array}$ & Pre: 0\% \\
Post: 9\%
\end{tabular}

Jornadas de Formación e Innovación Docente del Profesorado | № 2 (2019) Esta obra se distribuye con la licencia Creative Commons Reconocimiento-NoComercial-SinObraDerivada Internacional (CC BY-NC-ND 4.0.) 
Tabla 6. Pregunta 4 del cuestionario y niveles de aprendizaje: ¿Cómo podréis como geógrafas/os colaborar con vuestro entorno?

\begin{tabular}{|l|l|l|}
\hline $\begin{array}{l}\text { Nivel de } \\
\text { respuesta }\end{array}$ & Descripción & $\begin{array}{l}\text { Porcentaje de } \\
\text { respuestas }\end{array}$ \\
\hline Nivel 1 & No da una respuesta & $\begin{array}{l}\text { Pre: } 54 \% \\
\text { Post:18\% }\end{array}$ \\
\hline Nivel 2 & $\begin{array}{l}\text { Respuesta incompleta y no relacionada con } \\
\text { su entorno social o con la Geografía. }\end{array}$ & $\begin{array}{l}\text { Pre: 38\% } \\
\text { Post: 59\% }\end{array}$ \\
\hline Nivel 3 & $\begin{array}{l}\text { Nombra a la Administración o campos } \\
\text { aplicados de la Geografía pero sin contexto o } \\
\text { implicación social. }\end{array}$ & $\begin{array}{l}\text { Pre: 8\% } \\
\text { Post: 18\% }\end{array}$ \\
\hline Nivel $\mathbf{4}$ & $\begin{array}{l}\text { Utiliza ejemplos justificados, variados } \\
\text { y cercanos, relacionándolos con otros } \\
\text { conceptos como la exclusión o las } \\
\text { desigualdades. }\end{array}$ & $\begin{array}{l}\text { Pre: 0\% } \\
\text { Post: 5\% }\end{array}$ \\
\hline
\end{tabular}

Podemos concluir de los resultados que en el caso de las preguntas más relacionadas con los contenidos de la asignatura y el grado la mejora ha sido notable aunque era esperable teniendo en cuenta que se trata de alumnado de primer cuatrimestre de primero. Los avances han sido menores en la pregunta más relacionadas con actitudes a pesar de ser una metodología muy centrada en la aportación a la comunidad.

Para una evaluación global del aprendizaje del alumnado se podría agrupar las respuestas de las distintas preguntas a 4 posibles niveles de respuesta siguiendo este esquema:

Tabla 7. Niveles de respuesta evaluación global del aprendizaje.

\begin{tabular}{|l|l|}
\hline Nivel de respuesta & Descripción \\
\hline Nivel 1 & Respuestas incorrectas o no razonadas. \\
\hline Nivel 2 & Respuestas incompletas y no estructuradas. \\
\hline
\end{tabular}

Jornadas de Formación e Innovación Docente del Profesorado | № 2 (2019) Esta obra se distribuye con la licencia Creative Commons Reconocimiento-NoComercial-SinObraDerivada Internacional (CC BY-NC-ND 4.0.) 


\begin{tabular}{|l|l|}
\hline Nivel 3 & $\begin{array}{l}\text { Respuestas correctas pero no completas para lo } \\
\text { que se espera tras el el CIMA. }\end{array}$ \\
\hline Nivel 4 & $\begin{array}{l}\text { Respuestas más completas y cercanas a los } \\
\text { contenidos trabajados en el en el CIMA. }\end{array}$ \\
\hline
\end{tabular}

Con esta agrupación de respuestas podemos observar (figuras 2 y 3 ) que los porcentajes de contestaciones de los escalones inferiores, que se corresponden con los contenidos más básicos, se han reducido. Se ha producido un notable trasvase de respuestas desde el escalón inferior a los intermedios, y tras el ciclo de mejora aparecen también respuestas en el escalón superior, inexistentes al principio de la tarea.

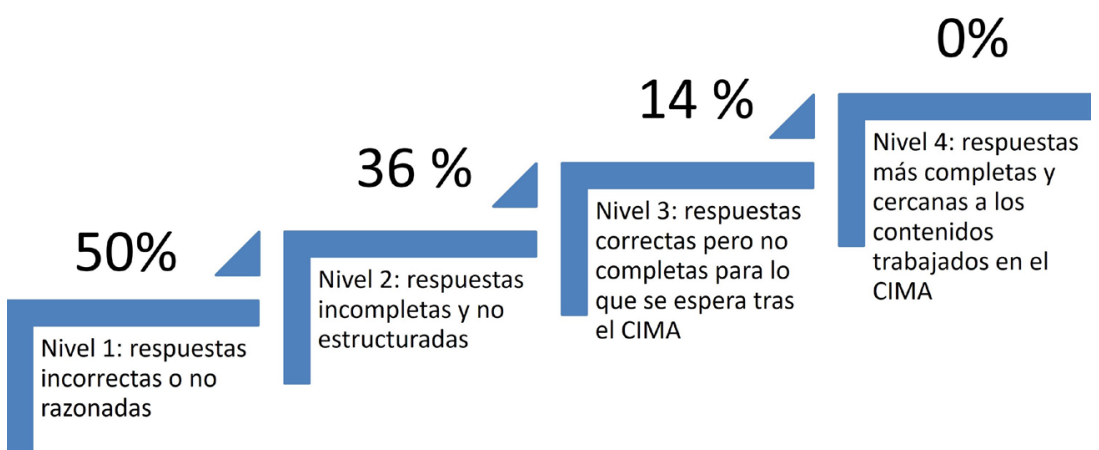

Figura 2: Porcentaje de respuestas de las distintas preguntas clasificadas por los 4 posibles niveles de respuesta en los cuestionarios previos.

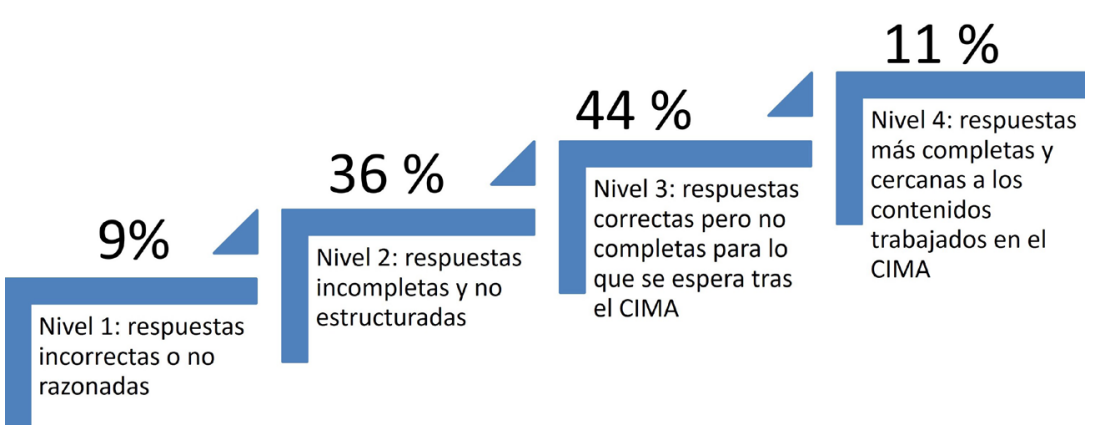

Figura 3: Porcentaje de respuestas de las distintas preguntas clasificadas por los 4 posibles niveles de respuesta en los cuestionarios finales.

Jornadas de Formación e Innovación Docente del Profesorado | № 2 (2019) Esta obra se distribuye con la licencia Creative Commons Reconocimiento-NoComercial-SinObraDerivada Internacional (CC BY-NC-ND 4.0.) 
Por último, se acordó que la evaluación se llevaría a cabo por medio de autoevaluación y la evaluación del docente siguiendo una rúbrica consensuada y que contuviese los criterios de evaluación de la asignatura tras la presentación final del último prototipo y un texto resumen o vídeo de corta duración explicativo del proceso llevado hasta la presentación del prototipo.

\section{Evaluación del CIMA.}

En cuanto a la evaluación del propio diseño del CIMA y de la intervención en el aula, como se ha comentado en anteriores apartados, se llevó a cabo un diario del profesor de las sesiones y se analizaron las respuestas a las preguntas realizadas a los alumnos en la última sesión a partir de las preguntas del cuestionario entregado para evaluar la metodología docente.

Tras las sesiones en el aula y el estudio de los cuestionarios, hay que destacar que uno de los aspectos que no han funcionado en la puesta en práctica del CIMA es la escasa presencia de la creatividad en retos y soluciones. Al ser también bastante limitante la fecha final de aplicación del CIMA, no hubo ocasión ni de crear prototipos en todos los grupos o segundas versiones en los grupos que sí llegaron a esa fase ni de llevar a cabo más aportaciones de ideas-soluciones. Además, la mayor parte de los criterios que los propios grupos escogían para evaluar sus ideas estaban relacionados con la accesibilidad, la universalidad y, especialmente, con el coste del desarrollo de las mismas. Olvidaron, por tanto, la originalidad y la creatividad en sus soluciones.

Otro de los problemas detectados está relacionado con la pérdida del foco de atención en la sociedad durante el proceso. Valoraron muy positivamente la fase de empatizar con otros colectivos con la realización de entrevistas 
pero en las fases más creativa del proceso volvían a poner el foco de atención en las inquietudes de los componentes del grupo y no tanto de los colectivos entrevistados, las personas a las que se estaban dirigiendo.

En cualquier caso, buena parte de estas dificultades tienen que ver con que sea esta la primera experiencia que tanto el alumnado como el docente tiene con esta metodología y, especialmente, la selección de una asignatura de primer cuatrimestre de primero. Uno de los alumnos lo destacaba en el cuestionario: "es un trabajo amplio y ambicioso para gente de primero de carrera". Otro grupo resumía esta dificultad en sus opiniones para la mejora: "realizar un trabajo abarcando tanta información estando los alumnos en primero, que no sabemos tanto".

Por todo ello, para futuras experiencias modificaría la secuenciación de las actividades para prolongar el tiempo de aplicación y favorecer una mayor temporalización de las sesiones dedicadas a dos momentos concretos del proceso. Por un lado, hay que aumentar las sesiones dedicadas a la determinación del reto (algunos comentarios del alumnado fueron: "el reto es muy ambiguo", "nos falta centrar más el campo de trabajo". Y por otro, hay que fomentar el debate en todo momento y especialmente en las sesiones finales, estudio de las soluciones y materiales generados por cada grupo, ya que es en este momento cuando el alumnado ha destacado una mayor valoración del trabajo en equipo, la colaboración y cercanía a su propia experiencia y futura situación profesional con comentarios como "lo que más me ha gustado del trabajo que estamos realizando es que cada persona ha podido elegir sobre qué hacer el trabajo, es decir, en lo que te quieres especializar en el futuro".

Entre los aspectos positivos destaco el trabajo cooperativo y los debates generados en la puesta común de retos, ideas y prototipos iniciales con muchos ejemplos de 
colaboración entre grupos. Algunos de los comentarios del alumnado van en esa línea: "es un trabajo muy dinámico, donde puedo escuchar las ideas de los demás grupos y dar una opinión acerca de ello", "me gustó el aspecto grupal y de colaboración porque pudimos compartir ideas y opiniones", "destaco el trabajo en grupo e interacciones con los demás alumnos" o este comentario de una alumna Erasmus "en Italia hay un método de trabajo muy teórico y aquí con esta metodología más práctica he conocido más cosas prácticas y reales y he entendido como se puede aplicar la Geografía en el mundo actual y la sociedad. He podido también entender las ideas de otros compañeros".

Para concluir, considero que los principios didácticos que tras esta experiencia deben permanecer en mi práctica docente futura son el uso de los retos y el trabajo colaborativo del alumnado y la necesidad de relacionar ese trabajo con el contexto social y personal del alumnado.

Jornadas de Formación e Innovación Docente del Profesorado | № 2 (2019) Esta obra se distribuye con la licencia Creative Commons 


\section{Referencias bibliográficas}

Bain, K. (2007). Lo que hacen los mejores profesores universitarios. Valencia: Publicaciones de la Universidad de Valencia.

Finkel, D. (2008). Dar clase con la boca cerrada. Valencia: Publicaciones de la Universidad de Valencia.

GOTIME (2017). ECO: Explora, Crea, Ofrece. Design Thinking para docentes. Recuperado de:

http://www.gotime.es

Porlán Ariza, R. (coord.) (2017). Enseñanza Universitaria. Cómo mejorarla. Madrid: Ediciones Morata.

Steinbeck, R. (2011). El «design thinking» como estrategia de creatividad en la distancia. Comunicar, 19(37), 27-35.

Torres-Gordillo, J. J., Camacho-Taboada, V. García-Jiménez, J., Herrero-Vázquez, E. A., Mora-Gutiérrez, J. P., y (2019). Aprendizaje como proceso de diseño: innovación universitaria desde el Design Thinking. Comunicación presentada a las I Jornadas de Innovación Docente y Proyectos Estudiantiles Vicerrectorado de Estudiantes. Universidad de Sevilla, del 29 al 31 de mayo 2019.

Jornadas de Formación e Innovación Docente del Profesorado | № 2 (2019) Esta obra se distribuye con la licencia Creative Commons Reconocimiento-NoComercial-SinObraDerivada Internacional (CC BY-NC-ND 4.0.) 